\title{
NUMERICAL METHODS FOR APPROXIMATING EIGENVALUES OF BOUNDARY VALUE PROBLEMS
}

\author{
KIAZ A. USMANI
Department of Applied Mathematics
University of Manitoba
Winnipeg, Manitoba R3T 2N2
Canada \\ and \\ MOHAMMAD ISA \\ College of Engineering \\ King Abdul Aziz University \\ P.0. Box 9027 \\ Jeddah, Saudi Arabia \\ (Recelved November 21, 1985)
}

\begin{abstract}
This paper describes some new finite difference methods for the approximation of eigenvalues of a two point boundary value problem associated with a fourth order linear differential equation of the type (py")" $-\left(q y^{\prime}\right)^{\prime}+(r-\lambda s) y=0$. The smallest positive eigenvalue of some typical eigensystems is computed to demonstrate the practical usefulness of the numerical techniques developed.
\end{abstract}

KEY WORDS AND PHRASES. Band matrices, Deflation, Finite-difference methods, Generalized matrix eigenvalue problem, Inverse power iteration, The Smallest eigenvalue of a matrix eigenvalue problem, Two-point boundary value problems.

1980 AMS SUBJECT CLASSIFICATION CODE. $65 \mathrm{~L} 15$.

1. INTRODUCTION. In this paper we will consider the fourth order linear differential equation

$$
L y \equiv\left[p(x) y^{\prime \prime}(x)\right]^{\prime \prime}-\left[q(x) y^{\prime}(x)\right]^{\prime}+[r(x)-\lambda s(x)] y(x)=0 \text {. }
$$

$a \leqq x \leqq b$, associated with one of the following pairs of homogeneous boundary conditions

$$
\begin{aligned}
& y(a)=y^{\prime \prime}(a)=y(b)=y^{\prime \prime}(b)=0 \\
& y(a)=y^{\prime}(a)=y(b)=y^{\prime}(b)=0
\end{aligned}
$$

Boundary value problems of the type (1.1)-(1.2) and/or (1.1)-(1.3) together with some of their modifications occur frequently in applied mathematics, modern physics and electrical engineering, see $[5,7,8,12]$. In (1.1), we assume that the real-valued functions $p(x), r(x)$ and $s(x)$ are continuous on $[a, b]$ and satisfy the conditions 
$p(x) \in c^{2}[a, b], q(x) \in C^{\prime}[a, b]$ and $p(x), q(x), s(x)>0, r(x) \geq 0, x \in[a, b$

Recently, numerical techniques of order 2 and 4 have been developed for computing approximate values of $\lambda$ for the boundary value problem (1.1-(1.3) with $p(x) \equiv 1$, $q(x) \equiv 0$, see $[1,2]$. In the fourth order method, the problem is discretized to yield a generalized seven-band symmetric matrix eigenvalue problem of the form

$$
A Y=\Lambda h^{4} B Y
$$

where $\Lambda$ is an approximate value of $\lambda$ with eigenvector $Y$ and $B$ is a diagonal matrix depending on the function $s(x)$. Consequently, the eigenvalue problem (1.5) can be converted to the usual standard problem of the type $M Y=\Lambda Y$ without any excessive amount of computational effort. There are at present several areas of research activity surrounding the development and analysis of numerical methods for approximating $\Lambda$ satisfying the generalized matrix eigenproblems of the type (1.5), see $[4,9,10,11,13]$.

Usmani has developed [14] some new finite difference methods of order 2 and 4 for computing eigenvalues of the differential system $(1.1)$ with $p(x) \equiv 1, q(x) \equiv 0$ (for $p(x) \equiv 0, q(x) \equiv 1$, see [15]) associated with the boundary conditions

$$
y(a)=y^{\prime}(a)=y^{\prime \prime}(b)=y^{\prime \prime}(b)=0
$$

The purpose of this work is to present some new finite difference methods for computing approximate values of $\lambda$ for the boundary value problems $(1.1)-(1.2)$ and $(1.1)-(1.3)$. These methods lead to generalized eigenvalue problems of the form (1.5) where $A$ is a five-band or seven-band matrix and $B$ is a diagonal positive definite matrix. We preface the numerical methods by some analytical properties of the eigenvalues and eigenfunctions of the boundary value problems under discussion.

2. PROPERTIES OF EIGENVALUES AND EIGENFUNCTIONS.

Let $\Pi$ stand for any of the two boundary value problems $(1.1)-(1.2)$ and (1.1)$(1.3)$.

THEOREM 2.1.

If $\lambda_{1}$ and $\lambda_{2}$ are two distinct eigenvalues of the problem II aand $y_{1}(x)$, $y_{2}(x)$ are the corresponding eigenfunctions, then

$$
\int_{a}^{b} s(x) y_{1}(x) y_{2}(x) d x=0
$$

PROOF. The proof is a direct consequence of Green's identity (see [3], p. 86) and the boundary conditions $(1.2)$ and (1.3).

LEMMA 2.2. If $y(x)$ is an eigenfunction belonging to the eigenvalue $\lambda$ of the boundary value problem $I$, then $\bar{y}(x)$ is an eigenfunction belonging to the eigenvalue $\bar{\lambda}$.

PROOF. The proof is trivial and follows from $\overline{L Y}=0$. 
THEOREM 2.3 .

The eigenvalues of the boundary value problem $\pi$, together with $(1.4)$, are real. In fact

$$
\lambda=\frac{\int_{a}^{b}\left[p\left(y^{\prime \prime}\right)^{2}+q\left(y^{\prime}\right)^{2}+r y^{2}\right] d x}{\int_{a}^{b} s y^{2} d x}>0 .
$$

PROOF. Let $\lambda=\mu+i \nu, \mu, \nu \in R$, be an eigenvalue of the problem II with eigenfunction $y(x)=u(x)+i v(x)$ where $u(x)$ and $v(x)$ are real-valued functions. From Lemma 2.2, it follows that $\bar{\lambda}$ is also an elgenvalue of the problem il with respect to the eigenfunction $\bar{y}(x)=u(x)-i v(x)$. Now, from Theorem 2.1, it follows that

$$
0=\int_{a}^{b} s(x) y(x) \bar{y}(x) d x=\int_{a}^{b} s(x)|y(x)|^{2} d x>0
$$

because $s(x)>0$ and $y(x) \neq 0$. The contradiction in (2.3) suggests that $\lambda$ cannot be complex, hence it is real as required.

In order to prove $(2.2)$, we multiply $(1.1)$ by $y(x)$ and integrate the resulting equation twice from $x=a$ to $x=b$. We consequently arrive at (2.2) on using the conditions $(1.1)-(1.4)$.

3. A SECOND ORDER METHOD FOR COMPUTING $\lambda$ FOR $(1.1)-(1.2)$.

For a positive integer $n \geqq 4$, let $h=\frac{b-a}{n+1}$ and $x_{1}=a+1 h, 1=0(1) n+1$. We shall also designate $y_{i}=y\left(x_{i}\right), p_{i}=p\left(x_{i}\right)$ etc. We discretize the boundary value problem $(1.1)-(1.2)$ by the following set of difference equations

(a) $\left[4 p_{1}+p_{2}+h^{2}\left(q_{1 / 2}+q_{3 / 2}\right)+h^{4} r_{1}\right] y_{1}-\left[2 p_{1}+2 p_{2}+h^{2} q_{3 / 2}\right] y_{2}+p_{2} y_{3}=$

$$
\lambda h^{4} s_{1} y_{1}+0\left(h^{4}\right)
$$

(b)

$$
\begin{aligned}
& p_{i-1} y_{i-2}-\left[2 p_{i-1}+2 p_{i+1}+h^{2} q_{i-1 / 2}\right] y_{i-1}+\left[p_{i-1}+4 p_{i}+p_{i+1}+h^{2}\left(q_{i-1 / 2}+\right.\right. \\
& \left.\left.q_{i+1 / 2}\right)+h^{4} r_{i}\right] y_{i}-\left[2 p_{i}+2 p_{i+1}+h^{2} q_{i+1 / 2}\right] y_{i+1}+p_{i+1} y_{i+2}=\lambda h^{4} s_{i} y_{i}+0\left(h^{6}\right), \\
& 1=2(1) n-1,
\end{aligned}
$$

(c) $p_{n-1} y_{n-2}-\left[2 p_{n-1}+2 p_{n}+h^{2} q_{n-1 / 2}\right] y_{n-1}+\left[p_{n-1}+4 p_{n}+h^{2}\left(q_{n-1 / 2}+q_{n+1 / 2}\right)\right.$

$\left.+h^{4} r_{n}\right] y_{n}=\lambda h^{4} s_{n} y_{n}+O\left(h^{4}\right)$

The difference equation (3.1b) os pbtaomed bu wrotomg $(1.1)$ at $x=x_{i}$, in the form

$$
h^{2}\left(p y^{\prime \prime}\right)_{i}^{\prime \prime}-h^{2}\left(q y^{\prime}\right)_{i}^{\prime}+h^{2}\left(r_{i}-\lambda s_{i}\right) y_{i}=0 \text {, }
$$




$$
\left(p y^{\prime \prime}\right)_{1-1}-2\left(p y^{\prime \prime}\right)_{1}+\left(p y^{\prime \prime}\right)_{1+1}-h\left[\left(q y^{\prime}\right)_{1+1 / 2}\left(q y^{\prime}\right)_{1-1 / 2}\right]+h^{2}\left(r_{1}-\lambda s_{1}\right) y_{1}=0\left(h^{4}\right),
$$

or

$$
\begin{aligned}
& p_{1-1}\left[y_{1-2}-2 y_{1-1}+y_{i}\right]-2 p_{1}\left[y_{1-1}-2 y_{1}+y_{i+1}\right] \\
& +p_{i+1}\left[y_{i}-2 y_{1-1}+y_{1+2}\right]-h^{2}\left[q_{1+1 / 2}\left(y_{1+1}-y_{i}\right)-q_{1-1 / 2}\left(y_{1}-y_{1-1}\right)\right] \\
& +h^{4}\left(r_{i}-\lambda s_{i}\right) y_{1}=0\left(h^{6}\right),
\end{aligned}
$$

which can be arranged in the desired form (3.1b). The difference equations (3.1a) and (3.1c) are introduced so that the resulting coefficient matrix in (3.1) is a five-band symmetric matrix. In order to obtain (3.1a), we write (1.1) at $x=x_{1}$ in the form

$$
\begin{aligned}
& \mathrm{h}^{2}\left(\mathrm{py} \mathrm{y}_{1}\right)_{1}-\mathrm{h}^{2}\left(q \mathrm{y}^{\prime}\right)_{1}^{\prime}+\mathrm{h}^{2}\left(\mathrm{r}_{1}-\lambda \mathrm{s}_{1}\right) \mathrm{y}_{1}=0, \\
& \mathrm{p}_{0} \mathrm{y}_{0}^{\prime \prime}-2 \mathrm{p}_{1} \mathrm{y}_{1}^{\prime \prime}+\mathrm{p}_{2} \mathrm{y}_{2}^{\prime \prime}-\mathrm{h}\left(\mathrm{q}_{3} \mathrm{2}_{2} \mathrm{y}_{3}^{\prime} /^{2}-\mathrm{q}_{1 / 2} \mathrm{y}_{1 / 2}^{\prime}\right)+\mathrm{h}^{2}\left(\mathrm{r}_{1}-\lambda s_{1}\right) \mathrm{y}_{1}= \\
& 0\left(\mathrm{~h}^{4}\right),
\end{aligned}
$$

or

$$
\begin{aligned}
& -2 p_{1}\left(y_{0}-2 y_{1}+y_{2}\right)+p_{2}\left(y_{1}-2 y_{2}+y_{3}\right)-h^{2}\left[q_{1 / 2}\left(y_{2}-y_{1}\right)\right. \\
& \left.-q_{1 / 2}\left(y_{1}-y_{0}\right)\right]+h^{4}\left(r_{1}-\lambda s_{1}\right) y_{1}=0\left(h^{4}\right) .
\end{aligned}
$$

The preceding equation is easily arranged in the form (3.1a). The difference equation (3.1c) is developed in an analogous manner by writing (1.1) at $x=x_{n}$. The system of linear equations (3.1) can be written in matrix form

$$
\left(A+h^{4} R\right) y=\lambda h^{4} S y+t(h)
$$

where $A$ is a symmetric five-band matrix. The matrices $R=\operatorname{diag}\left(r_{i}\right), S=\operatorname{diag}\left(s_{i}\right)$ are diagonal matrices and $y=\left[\begin{array}{lll}y_{1} & y_{2} \ldots y_{n}\end{array}\right]^{T} t(h)=\left[\begin{array}{lll}t_{1} & t_{2} \ldots t_{n}\end{array}\right]^{T}$. Here $t_{1}, t_{n}=0\left(h^{4}\right)$ and $t_{i}=0\left(h^{6}\right), i=2(1) n-1$.

Thus, our method for computing approximations $\Lambda$ for $\lambda$ satisfying (1.1)-(1.2) can be expressed as a generalized five-band symmetric matrix eigenvalue problem

$$
\left(A+h^{4} R\right) Y=\Lambda h^{4} S Y \text {. }
$$

It can be proved that $A$ is a positive definite matrix and hence for any stepsize $h>0$, the approximations $\Lambda$ for $\lambda$ by (3.5) are real and positive for all p, q, s $>0$ and $\mathbf{r} \geqq 0$. That our method provides $0\left(h^{2}\right)$ convergent approximations $\Lambda$ for $\lambda$ can be established following Grigorieff [6]. We omit the long and tedious details of convergence proof for brevity.

Normally, only one or a few of the extreme eigenvalues of $(1.1)-(1.2)$ are needed in applications. In what follows, we will compute only the smallest eigenvalue of the 
system to illustrate our method based on (3.1). We consider the eigenvalue problems

$$
\left[\left(1+x^{2}\right) y^{\prime \prime}\right]^{\prime \prime}-\left[\left(1+x^{2}\right) y^{\prime}\right]^{\prime}+\left[\frac{1}{(1+x)^{2}}-\lambda(1+x)^{4}\right] y=0
$$

with boundary conditions $(1.2)$ at $a=0, b=1$, and

$$
\left[e^{x} y^{\prime \prime}\right]^{\prime \prime}-\left[e^{x} y^{\prime}\right]^{\prime}+[\sin x-\lambda \cos x] y=0
$$

with boundary conditions (1.2) at $a=0, b=1$. We computed approximations to the smallest eigenbalue $\Lambda_{1}$ of these eigensystems by our method (3.1) or equivalently (3.5). The corresponding relative errors are shown in Table I. It is evident from the entries of the accompanying table that our numberical method provides $0\left(h^{2}\right)$ convergent approximations. In computing the relative errors, we assumed that $\lambda_{1}=\Lambda_{1}$ with $h=2^{-8}$, because exact value of $\lambda_{1}$ cannot be obtained by analytical methods for these eigensystems.

TABLE I

Observed relative errors for $h=2^{-m}, m=3(1) 7$

\begin{tabular}{llll}
\hline \multirow{2}{*}{ Problem } & \multicolumn{1}{c}{$\Lambda_{1}$} & Relative Error $\mid 1-\frac{\lambda_{1}}{\Lambda_{1}}$ \\
\hline \multirow{2}{*}{$(3.6)$} & $2^{-3}$ & $24.634,681$ & $2.448-2 *$ \\
& $2^{-4}$ & $25.085,489$ & $6.068-3$ \\
& $2^{-5}$ & $25.199,984$ & $1.497-3$ \\
& $2^{-6}$ & $25.228,721$ & $3.563-4$ \\
& $2^{-7}$ & $25.235,913$ & $7.125-5$ \\
& $2^{-8}$ & $25.237,711$ & 0.0 \\
\hline \multirow{2}{*}{$3.7)$} & $2^{-3}$ & $19.548,553$ & $2.551-2$ \\
& $2^{-4}$ & $19.921,847$ & $6.294-3$ \\
& $2^{-5}$ & $20.016,196$ & $1.551-3$ \\
& $2^{-6}$ & $20.039,847$ & $3.691-4$ \\
$2^{-7}$ & $20.045,764$ & $7.380-5$ \\
$2^{-8}$ & $20.047,244$ & 0.0 \\
\hline
\end{tabular}

* We write $2.448-2$ for $2.448 \times 10^{-2}$.

4. A METHOD FOR COMPUTING $\lambda$ FOR $(1.1)-(1.3)$.

We omit the lengthy details of the development of our numerical method but we remark that a second order method for computing approximations $\Lambda$ to $\lambda$ satisfying $(1.1)-(1.3)$ is based on

$$
\left(A^{\prime}+h^{4} R\right) Y=\Lambda h^{4} S Y
$$


where $A^{\prime}$ differs from A introduced in (3.5) in the first and the last rows only. The first row of $A^{\prime}$ is

$$
\left[\left\{2 p_{0}+4 p_{1}+p_{2}+h^{2}\left(q_{1 / 2}+q_{3 / 2}\right)\right\}-\left\{2 p_{1}+2 p_{2}+h^{2} q_{3 / 2}\right\} p_{2} 0 \ldots 0\right]
$$

and the last row of $A^{\prime}$ is

$\left[0 \ldots 0 p_{n-1}-\left\{2 p_{n-1}+2 p_{n}+h^{2} q_{n-1 / 2}\right\} \quad\left\{p_{n-1}+4 p_{n}+2 p_{n+1}+h^{2}\left(q_{n-1 / 2}+q_{n+1 / 2}\right)\right\}\right]$.

Again, as in the previous section, the matrix $A^{\prime}$ is a five-band symmetric matrix.

Another second order method for computing approximations $\Lambda$ to $\lambda$ satisfying $(1.1)-(1.3)$ is based on

$$
\left(A^{*}+h^{4} R\right) Y=\Lambda h^{4} S Y
$$

where $A^{\prime \prime}$, as before, differs from $A$ in the first and the last rows only. The first and the last rows of $A^{\prime \prime}$ are

$$
\left[\left\{4 p_{0}+4 p_{1}+p_{2}+h^{2}\left(q_{1 / 2}+q_{3 / 2}\right)\right\}-\left\{l / 2 p_{0}+2 p_{1}+2 p_{2}+h^{2} q_{3 / 2}\right\} p_{2} 0 \ldots 0\right]
$$

and

$\left[0 \ldots 0 p_{n-1}-\left\{2 p_{n-1}+2 p_{n}+1 / 2 p_{n+1}+h^{2} q_{n-1 / 2}\right\}\left\{p_{n+1}+4 p_{n}+4 p_{n-1}+h^{2}\left(q_{n-1 / 2}+q n+1 / 2\right)\right\}\right]$ respectively. The numerical results for computing $\Lambda$ based on (4.2) are slightly better than those based on (4.1), but the matrix $A^{\prime \prime}$ is no longer a symmetric matrix.

We illustrate our methods based on (4.1) and (4.2) by computing $\Lambda_{1}$ satisfying (3.7) and the boundary conditions (1.3) with $a=0$ and $b=1$.

The smallest eigenvalue $\Lambda_{1}$ is computed employing inverse power iteration method and the numerical results are summarized in Table II

Table II

Observed relative errors for Problem (3.7)-(1.3) with $a=0, b=1$

\begin{tabular}{llll}
\hline Method & \multicolumn{1}{c}{$\Lambda_{1}$} & Relative error \\
\hline \multirow{2}{*}{$(4.1)$} & $2^{-3}$ & 841.550 & $1.374-1$ \\
& $2^{-4}$ & 925.773 & $3.393-2$ \\
& $2^{-5}$ & 949.245 & $8.364-3$ \\
& $2^{-6}$ & 955.283 & $1.990-3$ \\
& $2^{-7}$ & 956.803 & $3.980-4$ \\
& $2^{-8}$ & 957.184 & 0.0 \\
\hline \multirow{2}{*}{ (4.2) } & $2^{-3}$ & 922.198 & $3.805-2$ \\
& $2^{-4}$ & 949.282 & $8.428-3$ \\
& $2^{-5}$ & 955.404 & $1.967-3$ \\
& $2^{-6}$ & 956.847 & $4.553-4$ \\
$2^{-7}$ & 957.197 & $8.983-5$ \\
$2^{-8}$ & 957.283 & 0.0
\end{tabular}


5. HIGHER ORDER METHODS FOR SPECIAL CASE OF $(1.1)$.

In this section we consider the linear differential equation

$$
y^{(4)}-q(x) y^{\prime \prime}+(r(x)-\lambda s(x)) y=0
$$

associated with the boundary conditions (1.2) or (1.3). For $n \geq 7$, let the stepsize $h$ and the sequence $\left\{x_{1}\right\}$ be defined as in section 3 .

Case 1. The boundary value problem $(5.1)-(1.2)$ is discretized by the following difference equations

$$
\begin{aligned}
& \text { (a) }\left(44+12 h^{2} q_{1}+6 h^{4} r_{1}\right) Y_{1}-\left(38+6 h^{4} q_{1}\right) Y_{2}+12 Y_{3}-Y_{4}=6 \Lambda h^{4} s_{1} Y_{1} \\
& \text { (b) }-\left(38+8 \mathrm{~h}^{2} \mathrm{q}_{2}\right) \mathrm{Y}_{1}+\left(56+15 \mathrm{~h}^{2} \mathrm{q}_{2}+6 \mathrm{~h}^{4} \mathrm{r}_{2}\right) \mathrm{Y}_{2}-\left(39+8 \mathrm{~h}^{2} \mathrm{q}_{2}\right) \mathrm{Y}_{3} \\
& +\left(12+\frac{h^{2}}{2} q_{2}\right) y_{4}-y_{5}=6 \Lambda h^{4} s_{2} y_{2}
\end{aligned}
$$

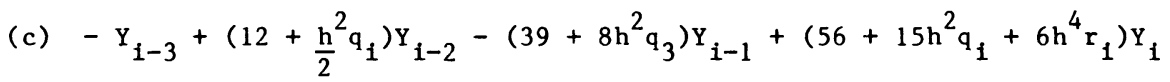

$$
\begin{aligned}
& -\left(39+8 h^{2} q_{i}\right) Y_{i+1}+\left(12+\frac{h^{2}}{2} q_{i}\right) Y_{i+2}-Y_{i+3}=6 \Lambda h^{4} s_{i} Y_{i}, i=3(1) n-2, \\
& \text { (d) }-Y_{n-4}+\left(12+\frac{h^{2}}{2} q_{n-1}\right) Y_{n-3}-\left(39+8 h^{2} q_{n-1}\right) Y_{n-2}+\left(56+15 h^{2} q_{n-1}+\right. \\
& \left.6 h^{4} r_{n-1}\right) Y_{n-1}-\left(38+8 h^{2} q_{n-1}\right) Y_{n}=6 \Lambda h^{4} s_{n-1} Y_{n-1} \text {, } \\
& \text { (e) }-Y_{n-3}+12 Y_{n-2}-\left(38+6 h^{2} q_{n}\right) Y_{n-1}+\left(44+12 h^{2} q_{n}+6 h^{4} r_{n}\right) Y_{n}=6 \Lambda h^{4} s_{n} Y_{n} \text {. }
\end{aligned}
$$

The system of equations (5.2) can be written in the form

$$
\left(A+h^{2} Q B+6 h^{4} R\right) Y=6 \Lambda h^{4} S Y
$$

where

$$
A=J^{3}+6 J^{2}, J=\left(j_{m n}\right) \text { is a tridiagonal matrix with } j_{m m}=2 \text {, }
$$

$j_{m, m+1}=-1$, and $B=\left(b_{i j}\right)$ is a five-band matrix so that

$$
\begin{gathered}
b_{11}=b_{n n}=12, b_{1,2}=b_{n, n-1}=6 \text { and } \\
b_{i j}=\left\{\begin{array}{rr}
15, & i=j \\
-8, & |i-j|=1 \\
1 / 2, & |i-j|=2 .
\end{array}\right\}
\end{gathered}
$$

Case 2. The boundary value problem (5.1)-(1.3) is discretized by the difference equations (see [1] also)

(a) $\left(76+2 h^{2} q_{0}+12 h^{2} q_{1}+6 h^{4} r_{1}\right) Y_{1}-\left(42+6 h^{2} q_{1}\right) Y_{2}+12 Y_{3}-Y_{4}=6 \Lambda h^{4} s_{1} Y_{1}$,

(b) $-\left(42+\frac{h^{2}}{2} q_{0}+8 h^{2} q_{2}\right) Y_{1}+\left(-\frac{113}{2}+15 h^{2} q_{2}+6 h^{4} r_{2}\right) Y_{2}-\left(39+8 h^{2} q_{2}\right) Y_{3}$

$+\left(12+\frac{h^{2}}{2} q_{2}\right) Y_{4}-Y_{5}=6 \Lambda h^{4} s_{2} Y_{2}$,

(c) The same equation as $(5.1 \mathrm{c}), \quad \mathrm{i}=3(1) \mathrm{n}-2$, 
(d) $-Y_{n-4}+\left(12+\frac{h^{2}}{2} q_{n-1}\right) Y_{n-3}-\left(39+8 h^{2} q_{n-1}\right) Y_{n-2}+\left(\frac{113}{2}+15 h^{2} q_{n-1}+\right.$ $\left.6 h^{4} r_{n-1}\right) Y_{n-1}-\left(42+8 h^{2} q_{n-1}+\frac{h^{2}}{2} q_{n+1}\right) Y_{n}=6 \Lambda h^{4} s_{n-1} Y_{n-1}$,

(e) $-Y_{n-3}+12 Y_{n-2}-\left(42+6 h^{2} q_{n}\right) Y_{n-1}+\left(76+12 h^{2} q_{n}+2 h^{2} q_{n+1}+6 h^{4} r_{n}\right) Y_{n}$

$=6 \Lambda h^{4} s_{n} Y_{n}$.

We computed approximations to $\Lambda_{1}$ for the boundary value problems $(5.1)-(1.2)$ and $(5.1)-(1.3)$ with $q(x)=1+x^{2}, r(x)=\frac{1}{(1+x)^{2}}, s(x)=(1+x)^{4}, a=0, b=1$. In both cases we assumed that $\lambda_{1}=\Lambda_{1}$ with $h=2^{-8}$. It is abundantly clear from the entries of the Table III that both our methods based on (5.2) and (5.4) are fourth order methods.

\section{TABLE III}

Observed relative errors $\left(0\left(h^{4}\right)\right.$-convergent numerical techniques)

$\begin{array}{llll}\text { Problem \& method } h & \Lambda_{1} & \text { Relative error }\end{array}$

\begin{tabular}{llll}
\hline $\begin{array}{l}\text { Problem (5.1)-(1.2) } \\
\text { based on method }\end{array}$ & $2^{-3}$ & $19.807,299$ & $7.203-4$ \\
$(5.2)$ & $2^{-4}$ & $19.820,723$ & $4.256-5$ \\
& $2^{-5}$ & $19.821,518$ & $2.469-6$ \\
& $2^{-6}$ & $19.821,564$ & $1.324-7$ \\
& $2^{-7}$ & $19.821,567$ & $5.515-8$ \\
& $2^{-8}$ & 19.821 .567 & 0.0 \\
\hline $\begin{array}{l}\text { Problem (5.1)-(1.3) } \\
\text { based on method }\end{array}$ & $2^{-3}$ & $93.932,101$ & $3.772-3$ \\
(5.4) & $2^{-4}$ & $94.265,002$ & $2.268-4$ \\
& $2^{-5}$ & $94.285,062$ & $1.398-5$ \\
& $2^{-6}$ & $94.286,299$ & $8.632-7$ \\
& $2^{-7}$ & $94.286,376$ & $4.800-8$ \\
\hline & $2^{-8}$ & $94.286,380$ & 0.0 \\
\hline
\end{tabular}

ACKNOWLEDGEMENT: This work was supported in part by a grant from NSERC of Canada. The author also wishes to thank Mr. Manzoor Hussain for the numerical implementation of the methods developed in this paper. 
REFERENCES

1. CHAWLA, M.M. A New Fourth order Finite Difference Method for Computing Eigenvalues of Fourth order Two Point Boundary Value Problems, IMA J. of Numerical Analysis, 3 (1983), 291-293.

2. CHAWLA, M.M. and KATTI, C.P. A New Symmetric Five Diagonal Finite Difference Method for Computing Eigenvalues of Fourth Order Two Point Boundary Value Problem, J. Computational and Applied Maths., 8 (1982), 135-136.

3. CODDINGTON, E.A. and LEVINSON, N. Theory of Ordinary Differential Equations, McGraw-Hi11 Book Company, New York, 1955.

4. EDWARDS, Brunce. Computation of Eigenvalues Using Two Point Boundary Value Problem Codes, Internat. J. Numer. Methods Engrg., 18 (1982), 1736-1738.

5. FOX, L. The Numerical Solution of Two Point Boundary Value Problems in Ordinary Differential Equations, Oxford University Press, Oxford, 1957.

6. GRIGORIEFF, R.D. Diskrete Approximation von Eigenwertproblemen II, Numerische Mathematik, 24 (1975), 415-433.

7. HILDEBRAND, F.B. Methods of Applied Mathematics, Prentice-Ha11, Engelwood Cliffs, N.J., 1952 .

8. HILDEBRAND, F.B. Advanced Calculus for Applications, Prentic-Hall, Engelwood, C1iff, N.J., 1964 .

9. KELLER, H.B. On the Accuracy of Finite Difference Approximations to the Eigenvalues of Differential and Integral Operators, Numer. Math., $\underline{7}$ (1965), 412-419.

10. KELLER, H.B. Numerical Methods for Two Point Boundary Value Problems, Blaisdel1, Waltham, Massachusetts, 1968.

11. MCCORMICK, STEPHEN F. A mesh refinement method for $\mathrm{AX}=\Lambda \mathrm{BX}$, Math. Comp. 36 (1981), 485-498.

12. REISS, E. J., CALLEGARI, A.J. and AHLUWALIA, D.S. Ordinary Differential Equations with Applications, Holt, Rinehart and Winston, New York, 1976.

13. TURYN, Lawrence. Perturbation of Two Point Boundary Value Problems with Eigenvalue Parameter in the Boundary Conditions, Proc. Roy. Soc. Edinburgh Section A, 94 (1983), 213-219.

14. USMANI, Riaz A. Finite Difference Methods for Computing Eigenvalues of Fourth Order Boundary Value Problems, Int. J. Math. and Mathematical Sciences, 9 (1986), 137-143.

15. USMANI, Riaz A. Some New Finite Difference Methods for Computing Eigenvalues of Two Point Boundary Value Problems, Computers and Maths. with Applications, 11 (1985), 903-909. 


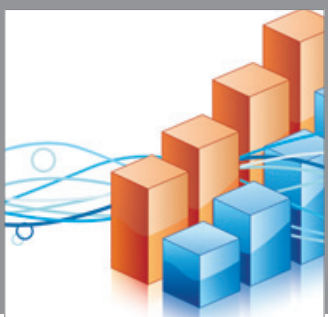

Advances in

Operations Research

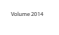

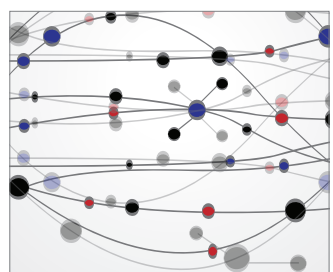

\section{The Scientific} World Journal
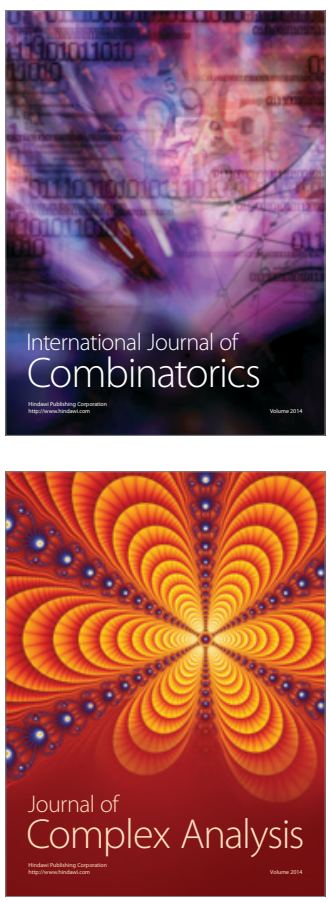

International Journal of

Mathematics and

Mathematical

Sciences
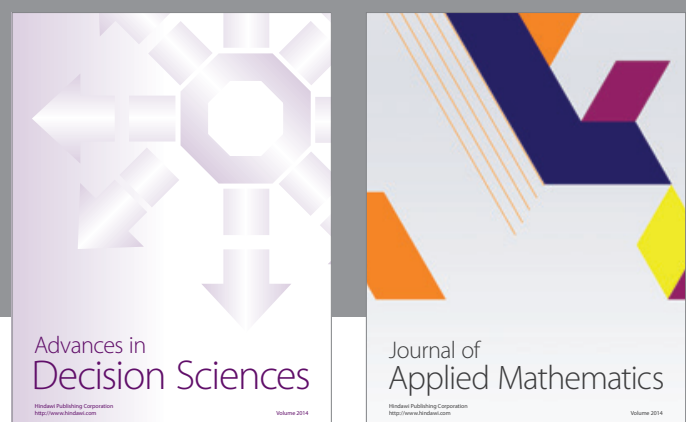

Journal of

Applied Mathematics
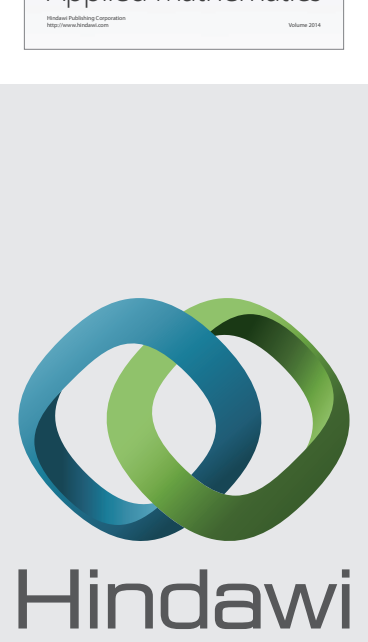

Submit your manuscripts at http://www.hindawi.com
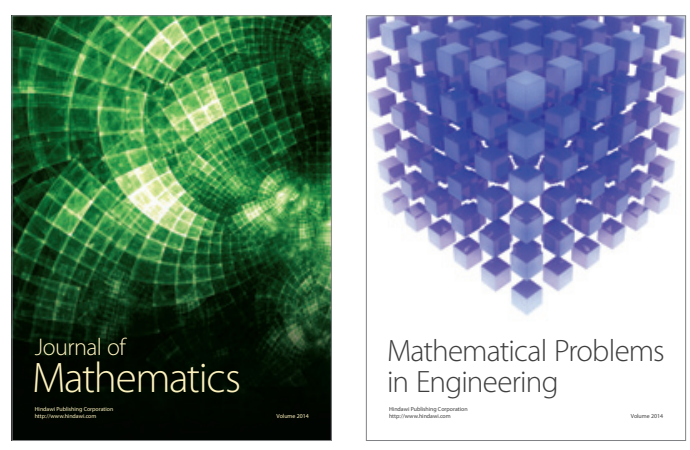

Mathematical Problems in Engineering
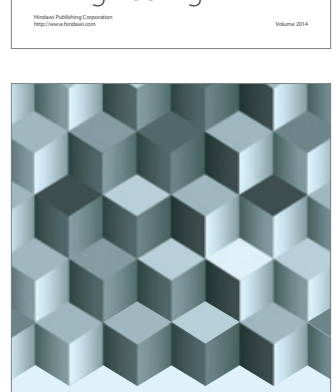

Journal of

Function Spaces
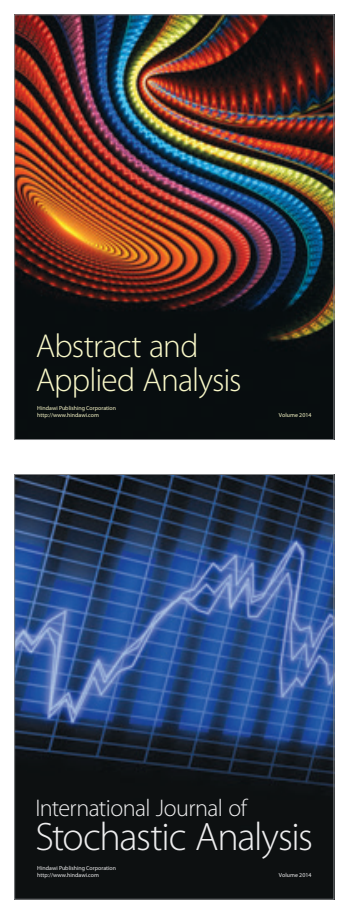

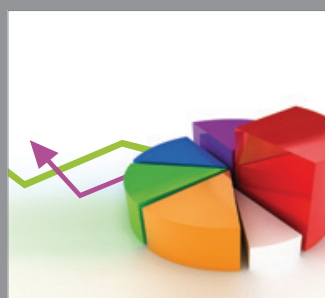

ournal of

Probability and Statistics

Promensencen
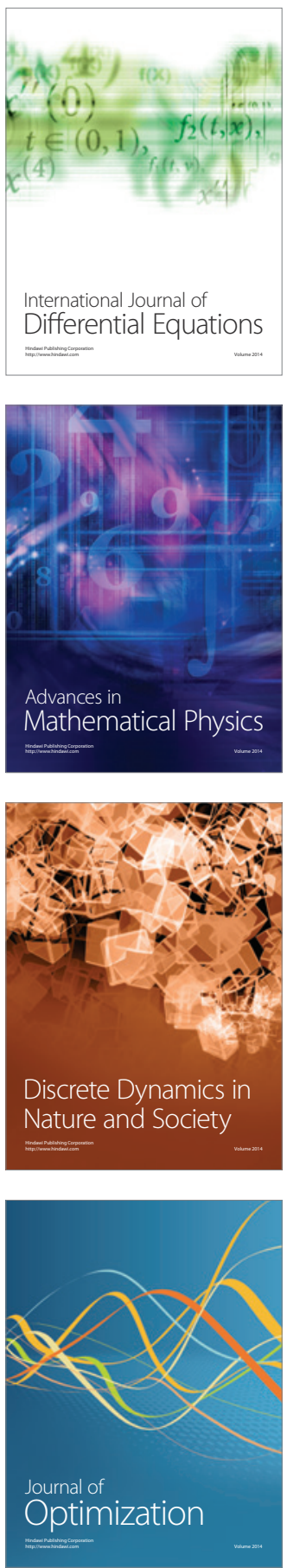